\title{
IgM Phospholipid Unit
}

National Cancer Institute

\section{Source}

National Cancer Institute. IgM Phospholipid Unit. NCI Thesaurus. Code C67348.

A unit for semiquantitative measurement of Ig M autoantibodies to proteins associated with negatively charged phospholipids evaluated in ELISA serum anticardiolipin or anionic phospholipid assay against an established reference standard. One Ig M phospholipid unit is equivalent to one $\mathrm{mg} / \mathrm{mL}$ of an affinity-purified standard IgM sample obtained from the specified individuals. 